\title{
The Immunotyping Distribution of Serum Monoclonal Paraprotein and Environmental Impact on Multiple Myeloma (MM) and Monoclonal Gammopathy of Uncertain Significance (MGUS) in Taiwan: A Medical Center-Based Experience
}

\author{
Chih-Chun Chang ${ }^{1 \&}$, Ming-Jang Su${ }^{1 \&}$, Shu-Jene Lee ${ }^{1 \&}$, Yu-Hui Tsai ${ }^{1}$, Lin-Yin \\ Kuo $^{1}$, I-Hsin Lin ${ }^{1}$, Hui-Ling Huang1, Tzung-Hai Yen ${ }^{2}$, Fang-Yeh Chu ${ }^{1,3 *}$
}

\begin{abstract}
Background: Whether ambient exposure to environmental pollutants leads to hematopoietic malignancies such as multiple myeloma (MM) remains to be ascertained. Therefore, we aimed to investigate the immunotyping distribution of serum monoclonal paraprotein and the environmental influence on MM and monoclonal gammopathy of uncertain significance (MGUS) in the Taiwanese population. Materials and Methods: Serum protein electrophoresis with immunosubtraction by the capillary zone electrophoresis method was performed as primary screening for MM and MGUS. Clinical, pathological, and residence data of patients were also obtained. Results: From August, 2013 to June, 2015, a total of 327 patients underwent serum protein electrophoresis with immunosubtraction. Among these, 281 demonstrated no remarkable findings or non-malignant oligoclonal gammopathy, 23 were detected to have MGUS, 18 were identified as MM, and a further 5 were found as other malignancies. The most frequent immunotyping distribution of serum monoclonal paraprotein was IgG kappa $(54.3 \%, n=25)$, followed by IgA lambda $(15.2 \%, n=7)$ and IgG lambda $(10.9 \%, n=5)$ in subjects with gammopathy. Additionally, it was shown that the elderly (OR: $4.61,95 \%$ CI: 1.88-11.30, $P<0.01)$ and males $($ OR: $2.04,95 \%$ CI: 1.04-4.02, $P=0.04)$ had significantly higher risk of developing MM and MGUS. There was no obvious impact of environmental factors on the health risk of MM and MGUS evolution (OR: 0.77, 95\% CI: 0.40-1.50, $P=0.49$ ). Conclusions: The most frequent immunotyping distribution of serum monoclonal paraprotein included IgG kappa, IgA lambda and IgG lambda in MM and MGUS in the Taiwanese population. The elderly and male subjects are at significantly higher risk of MM and MGUS development, but there was no obvious impact of environmental factors on risk.
\end{abstract}

Keywords: Multiple myeloma - monoclonal gammopathy of uncertain significance - protein electrophoresis

Asian Pac J Cancer Prev, 17 (1), 395-399

\section{Introduction}

Multiple myeloma (MM), one of the most frequent hematopoietic malignancies worldwide, is characterized by aberrant proliferation of monoclonal plasma cells (Rajabli et al., 2013; Sutandyo et al., 2015). In addition to biopsy-proven bony or extramedullary plasmacytoma, MM is often symptomatic and accompanied with plasma cell proliferation-related end organ damage like anemia, renal insufficiency and bony lesions (Rajkumar et al., 2014). Monoclonal gammopathy of undetermined significance (MGUS), instead, is clinically asymptomatic with few infiltrates of plasma cell in the bone marrow and the presence of monoclonal protein in serum (Kyle et al., 2010). MGUS is considered premalignant and needs long-term monitoring in prevention of disease evolution to malignant situations. Currently, serum protein electrophoresis with immunosubtraction assay has been the most common and initial screening panel for detection of MM and MGUS in clinical laboratories.

Recently, living environment and life quality have become essential issues as they significantly affect global health. According to the literature review, environmental exposure played an important role in increasing the risk and leading to poor prognosis of various disorders, including cancer, respiratory tract disorders, cardiovascular diseases, cerebrovascular accident and chronic renal failure (Tsai et al., 2003; Miller et al., 2007; Huang et al., 2013; Lin et al., 2015; Pan et al., 2015). It was also indicated that inhabitants' exposure to atmospheric

${ }^{1}$ Department of Clinical Pathology, Far Eastern Memorial Hospital, New Taipei City, ${ }^{2}$ Division of Nephrology and Clinical Toxicology, Chang Gung Memorial Hospital, Lin-Kou Medical Center, Taoyuan County, ${ }^{3}$ School of Medical Laboratory Science and Biotechnology, Taipei Medical University, Taipei City, Taiwan ${ }^{\circledR}$ Equal contributors *For correspondence: jacpha@mail.femh.org.tw 
carcinogenic substances was associated with the disparity of living districts (Huang et al., 2013; Chang et al., 2015). However, the environmental influence on hematopoietic malignancies such as MM remains to be ascertained.

Here, we conducted a retrospective study on analyzing the immunotyping distribution of serum monoclonal paraprotein in MM and MGUS as well as the environmental impact on the prevalence and immunotyping frequency of MM and MGUS in the Taiwanese population.

\section{Materials and Methods}

During the period of August, 2013 to June, 2015, patients who visited Far Eastern Memorial Hospital (FEMH), New Taipei City, Taiwan, and had available data of serum protein electrophoresis with immunosubtraction, were registered in the study. The laboratory data of serum protein electrophoresis with immunosubtraction was obtained retrospectively via the clinical biochemistry laboratory information management system (Technidata, TD-synergy). The protein electrophoresis and immunosubtraction of $\mathrm{IgG}$, IgA, IgM, kappa and lambda light chains were performed using the capillary zone electrophoresis method, on the instrument of CAPILLARYS 2 (software version 8.6.3, Bio-Check Inc., France) according to the manufacturer's maneuver (Gay-Bellile et al., 2003; Yang et al., 2007). Furthermore, the clinical data of patients who received serum protein electrophoresis with immunosubtraction were collected via the electronic medical chart review, including patient age, gender, living area (urban and suburban zones), clinical symptoms (such as bony pain or fracture, recurrent infections, anemic status and renal insufficiency), laboratory evidence (such as decreased hemoglobin concentration, elevated serum creatinine, calcium and lactate dehydrogenase levels), image study (such as lytic bone lesions), and final diagnosis of MGUS or MM with bone marrow biopsy-proved evidence. The stage of MM was determined based on the international staging system (ISS). Also, the data of environmental indicators in urban and suburban zones were adopted in our previous study for further analysis (Chang et al., 2015).

Unconditional logistic regression model was performed for the calculation of odds ratio (OR) to identify possible risk factors and to evaluate the effect of living area on patients with MM and MGUS, with the estimation of $95 \%$ confidence interval (CI). Data were presented as either mean \pm standard deviation (SD), number (percentage), or OR (lower-higher limits of 95\% CI). All statistical analyses were performed by the statistical software SPSS (version 15.0; SPSS Inc., Chicago, USA). A $P$ value less than 0.05 was considered to be statistically significant.

\section{Results}

Blood specimens were obtained from a total of 327 patients for serum protein electrophoresis with immunosubtraction. Among these patients, 281 were found no remarkable findings or non-malignant oligoclonal gammopathy and 46 were identified to have monoclonal gammopathy (Table 1). Of these 46 patients, 23 were identified as MGUS, 18 were identified as MM, and still other 5 were found as other malignancies on the basis of clinical symptoms, laboratory evidence and bone marrow biopsy. Among the cases with monoclonal gammopathy, the most frequent immunotyping of serum monoclonal paraprotein was IgG kappa $(54.3 \%, \mathrm{n}=25)$, followed by IgA lambda (15.2\%, n=7), IgG lambda $(10.9 \%, n=5)$,

Table 1. The demographic features in subjects underwent serum protein electrophoresis with immunosubtraction

\begin{tabular}{lc}
\hline Variables & Value or number $(\%)$ \\
\hline Age (year) & $71.7 \pm 13.7$ \\
Gender (Male/Female) & $158 / 169$ \\
Living environment (Urban/Suburban) & $202 / 125$ \\
Disease categorization & \\
No gammopathy / non-malignant oligocloncal gammopathy & $281(85.9 \%)$ \\
Monoclonal gammopathy & \\
MGUS & $23(8.0 \%)$ \\
MM & $18(5.5 \%)$ \\
stage 1 & $4(22.2 \%)$ \\
stage 2 & $6(33.3 \%)$ \\
stage 3 & $8(44.4 \%)$ \\
Other malignancies & $5(1.5 \%)$ \\
The immunotyping distribution of serum monoclonal paraprotein by serum protein electrophoresis with immunosubtraction. \\
IgG kappa & $25(54.3 \%)$ \\
IgA lambda & $7(15.2 \%)$ \\
IgG lambda & $5(10.9 \%)$ \\
Free kappa & $2(4.3 \%)$ \\
Free lambda & $2(4.3 \%)$ \\
IgA kappa & $1(2.2 \%)$ \\
IgM kappa & $1(2.2 \%)$ \\
IgM lambda & $1(2.2 \%)$ \\
IgG lambda \& IgA kappa & $1(2.2 \%)$ \\
IgG lambda \& free lambda & $1(2.2 \%)$ \\
\hline
\end{tabular}

Data were presented as either mean \pm standard deviation or number (percentage). MGUS, monoclonal gammopathy of uncertain significance; MM, multiple myeloma 
Immunotyping Distribution of Serum Monoclonal Paraprotein and Impact on Multiple Myeloma (MM) in Taiwan

free kappa light chain $(4.3 \%, \mathrm{n}=2)$, and free lambda light chain $(4.3 \%, n=2)$ (Table 1$)$. Of the cases with MGUS, the immunotyping of serum monoclonal paraprotein with IgG kappa (69.6\%, $\mathrm{n}=16)$, IgA lambda $(13.0 \%, \mathrm{n}=3)$, and IgG lambda $(8.7 \%, \mathrm{n}=2)$ were identified. Individuals who had immunotyping of serum monoclonal paraprotein with IgA kappa and biclonal gammopathy with IgG lambda and IgA kappa were also found (Table 2). Whereas in those subjects with MM, the most common immunotyping of serum monoclonal paraprotein was IgG kappa (38.9\%, $\mathrm{n}=7)$, followed by IgG lambda $(16.7 \%, \mathrm{n}=3)$, IgA lambda $(16.7 \%, n=3)$, free kappa light chain $(11.1 \%, n=2)$ and free lambda light chain $(11.1 \%, n=2)$. A case of MM who had biclonal gammopathy with immunotyping of serum monoclonal paraprotein with IgG lambda and free lambda light chain was identified as well (Table 2).

Univariate analysis revealed that the elders and male gender had significantly higher risk of the evolution of MM and MGUS (Table 3). In subjects with age more than 60 years old, the OR for MM and MGUS was 4.61 with $95 \%$ CI of $1.88-11.30(P<0.01)$, and the OR for $\mathrm{MM}$ was 5.82 with $95 \% \mathrm{CI}$ of $1.31-25.75(P<0.01)$ in comparison with those younger than 60 years old. In male subjects, the OR for MM and MGUS was 2.04 with $95 \%$

Table 2. The Immunotyping Distribution of Monoclonal Gammopathy of Uncertain Significance and Multiple Myeloma by serum Protein Electrophoresis with Immunosubtraction

\begin{tabular}{lc}
\hline Variables & Number $(\%)$ \\
\hline MGUS & \\
IgG kappa & $16(69.6 \%)$ \\
IgA lambda & $3(13.0 \%)$ \\
IgG lambda & $2(8.7 \%)$ \\
IgA kappa & $1(4.3 \%)$ \\
IgG lambda \& IgA kappa & $1(4.3 \%)$ \\
MM & \\
IgG kappa & $7(38.9 \%)$ \\
IgG lambda & $3(16.7 \%)$ \\
IgA lambda & $3(16.7 \%)$ \\
Free kappa & $2(11.1 \%)$ \\
Free lambda & $2(11.1 \%)$ \\
IgG lambda \& free lambda & $1(5.6 \%)$ \\
\hline
\end{tabular}

MGUS, monoclonal gammopathy of uncertain significance; MM, multiple myeloma
CI of 1.04-4.02 $(P=0.04)$, and the OR for MM was 1.75 with $95 \% \mathrm{CI}$ of $0.66-4.62(P=0.33)$ when compared with female gender. Additionally, it seemed that there was no apparent influence of environmental factors on the risk of developing MM and MGUS (Table 3). In those living in urban zones, the OR for MM and MGUS was 0.77 with 95\% CI of $0.40-1.50(P=0.49)$, and the OR for MM was 0.77 with $95 \% \mathrm{CI}$ of $0.30-2.01(P=0.62)$ in comparison with those living in suburban zones.

\section{Discussion}

In the present study, we investigated the immunotyping distribution of serum monoclonal paraprotein in MM and MGUS by the performance of serum protein capillary zone electrophoresis with the immunosubtraction method in the Taiwanese population. Our study indicated that IgG kappa, IgA lambda and IgG lambda were the most frequent immunotyping of serum monoclonal paraprotein in MM and MGUS. Besides, the elders and male subjects had significantly higher risk of the development of MM and MGUS. There was no apparent association of environmental factors with the risk of developing MM and MGUS.

Most MM patients were found to have a plasma cell-secreted paraprotein which could be presented as a restricted peak in the gamma zone by serum protein electrophoresis. Rarely, biclonal gammopathy was detected as the presence of two narrow peaks of paraprotein in the gamma region. According to the literature review, the most frequent immunotyping of paraprotein was IgG kappa (34\%), followed by IgG lambda (18\%), IgA kappa (13\%), free kappa light chain (9\%), IgA lambda (8\%) and free lambda light chain (7\%) in MM. Infrequently seen were biclonal $(2 \%), \operatorname{IgD}(2 \%)$ and $\operatorname{IgM}(0.5 \%)$ gammopathies (Kyle et al., 2003). Contrarily, patients with MGUS were usually asymptomatic, and the serum paraprotein at a concentration less than $3 \mathrm{~g} / \mathrm{dL}$ could be detected by protein electrophoresis. The monoclonal plasma cells could be found less than $10 \%$ in the bone marrow of MGUS subjects. However, patients with MGUS still needed to be monitored in the long term as there was approximately $1 \%$ annual accumulating risk of progression to MM (Kyle et al., 2007). The non-IgM and light chain immunotyping were the most commonly seen in MGUS (Rajkumar et al., 2010).

Table 3. Univariate Analysis for the Relationship of Patient Age, Gender and Living Environment to Monoclonal Gammopathy of Uncertain Significance and Multiple Myeloma

\begin{tabular}{|c|c|c|c|c|c|c|c|c|}
\hline & $\begin{array}{l}\text { No gammopathy } \\
\text { /oligoclonal } \\
\text { gammopathy }\end{array}$ & $\begin{array}{c}\text { MGUS } \\
\text { /MM }\end{array}$ & $\begin{array}{r}\text { Crude OR } \\
(95 \% \mathrm{CI})\end{array}$ & $P$ value & $\begin{array}{c}\text { No gammopathy } \\
\text { /oligoclonal } \\
\text { gammopathy / MGUS }\end{array}$ & MM & $\begin{array}{r}\text { Crude OR } \\
(95 \% \mathrm{CI})\end{array}$ & $P$ value \\
\hline \multicolumn{9}{|c|}{ Patient age (year) } \\
\hline$\geq 60$ & 157 & 35 & $4.61(1.88-11.30)$ & $<0.01$ & 176 & 16 & $5.82(1.31-25.75)$ & $<0.01$ \\
\hline$<60$ & 124 & 6 & & & 128 & 2 & & \\
\hline \multicolumn{9}{|l|}{ Gender } \\
\hline Male & 129 & 26 & $2.04(1.04-4.02)$ & 0.04 & 144 & 11 & $1.75(0.66-4.62)$ & 0.33 \\
\hline Female & 152 & 15 & & & 160 & 7 & & \\
\hline \multicolumn{9}{|l|}{ Living area } \\
\hline Urban & 175 & 23 & $0.77(0.40-1.50)$ & 0.49 & 188 & 10 & $0.77(0.30-2.01)$ & 0.62 \\
\hline Suburban & 106 & 18 & & & 116 & 8 & & \\
\hline
\end{tabular}


In many metropolitan areas in Asia, urban air pollution by mobile transport remains a major problem affecting public health (Chen et al., 2015). Subsequently, the underlying mechanism of environmental pollutants leading to tumorigenesis has been explored. It was disclosed that increased DNA strand breaks, reduced DNA repair capacity and DNA methylation were found in the population living in major cities, which potentially increased the health risk of cancer evolution (Ruchirawat et al., 2007; Cao et al., 2015). It was also revealed that certain gene mutation and modulation of microRNAs were associated with air pollution-related lung malignancies (Pan et al., 2015; Yu et al., 2015). Clinically, it was also reported that atmospheric exposure to air pollutants increased the health risk of non-respiratory malignancies, including breast cancer, hepatic cancer, gastric cancer, prostate cancer, bladder cancer and gynecological malignancies (Chiu et al., 2011; Al-Ahmadi et al., 2013; Hashim et al., 2014; Kakugawa et al., 2015). In recent study, it was indicated that long-term exposure to air contamination in outdoor was highly associated with acute myeloid leukemia (Linet et al., 2015), but not for other subtypes of leukemia such as chronic lymphocytic leukemia (Winters et al., 2015). Besides, it was shown that ambient exposure to carcinogenic air toxics restrictedly affected cancer-related public health. Similarly, no obvious association between environmental factors and development of MM and MGUS was indicated in our results. Despite no statistical significance, it was seemingly that there was lower risk of MM and MGUS development in the urban population. This could be partially explained by the high popularity of health examination and great accessibility to hospital care in the metropolitan area.Also, it was sometimes difficult to clarify the kind and source of carcinogenic pollutants, resulting in the complexity in evaluating environmental impact on public health.

The major limitations of the present study were the retrospective study design as well as the limited subject number. Besides, the follow-up of study population could not be long enough in the monitoring of disease progression and patient outcome due to environmental factors. Although accumulating evidence indicated that urban air contamination could affect the public health and potentially increase the cancer-related health risk, some studies indicated that effect of ambient exposure to certain noxious agents on the inhabitants was limited. The environmental influence on tumorigenesis remains controversial and further study is needed for clarifying the environmental impact on the evolution of pre- and malignant hematopoietic disorders, including MM and MGUS.

In conclusion, the most frequent immunotyping distribution of serum monoclonal paraprotein included IgG kappa, IgA lambda and IgG lambda in MM and MGUS in the Taiwanese population. The elders and male subjects had significantly higher risk of MM and MGUS evolution, but there was no apparent influence of environmental factors on the health risk of developing MM and MGUS.

\section{References}

Al-Ahmadi K, Al-Zahrani A (2013). NO (2) and cancer incidence in Saudi Arabia. Int J Environ Res Public Health, 10, 584462.

Cao Y (2015). Environmental pollution and DNA methylation: carcinogenesis, clinical significance, and practical applications. Front Med, 9, 261-74.

Chang CC, Chung YH, Liou CB, et al (2015). Influence of residential environment and lifestyle on multiple primary malignancies in Taiwan. Asian Pac J Cancer Prev, 16, 3533-8.

Chen G, Wan X, Yang G, et al (2015). Traffic-related air pollution and lung cancer: A meta-analysis. Thorac Cancer, 6, 307-18.

Chiu HF, Tsai SS, Chen PS, et al (2011). Traffic air pollution and risk of death from gastric cancer in Taiwan: petrol station density as an indicator of air pollutant exposure. $J$ Toxicol Environ Health A, 74, 1215-24.

Gay-Bellile C, Bengoufa D, Houze $P$, et al (2003). Automated multicapillary electrophoresis for analysis of human serum proteins. Clin Chem, 49, 1909-15.

Hashim D, Boffetta $P$ (2014). Occupational and environmental exposures and cancers in developing countries. Ann Glob Health, 80, 393-411.

Huang WH, Lin JL, Lin-Tan DT, et al (2013). Impact of living environment on 2-year mortality in elderly maintenance hemodialysis patients. PLoS One, 8, 74358.

Kakugawa Y, Kawai M, Nishino Y, et al (2015). Smoking and survival after breast cancer diagnosis in Japanese women: A prospective cohort study. Cancer Sci, 106, 1066-74.

Kyle RA, Gertz MA, Witzig TE, et al (2003). Review of 1027 patients with newly diagnosed multiple myeloma. Mayo Clin Proc, 78, 21-33.

Kyle RA, Remstein ED, Therneau TM, et al (2007). Clinical course and prognosis of smoldering (asymptomatic) multiple myeloma. $N$ Engl J Med, 356, 2582-90.

Kyle RA, Durie BGM, Rajkumar SV, et al (2010). Monoclonal gammopathy of undetermined significance (MGUS) and smoldering (asymptomatic) multiple myeloma: IMWG consensus perspectives risk factors for progression and guidelines for monitoring and management. Leukemia, 24, 1121-7.

Lin JH, Yen TH, Weng CH, et al (2015). Environmental $\mathrm{NO} 2$ level is associated with 2-year mortality in patients undergoing peritoneal dialysis. Med (Baltimore), 94, e368.

Linet MS, Yin SN, Gilbert ES, et al (2015). A retrospective cohort study of cause-specific mortality and incidence of hematopoietic malignancies in Chinese benzene-exposed workers. Int J Cancer, 137, 2184-97.

Miller KA, Siscovick DS, Sheppard L, et al (2007). Long-term exposure to air pollution and incidence of cardiovascular events in women. $N$ Engl J Med, 356, 447-58.

Pan HL, Wen ZS, Huang YC, et al (2015). Down-regulation of microRNA-144 in air pollution-related lung cancer. Sci Rep, 5, 14331.

Rajabli N, Naeimi-Tabeie M, Jahangirrad A, et al (2013). Epidemiology of leukemia and multiple myeloma in Golestan, Iran. Asian Pac J Cancer Prev, 14, 2333-6.

Rajkumar SV, Kyle RA, Buadi FK, et al (2010). Advances in the diagnosis, classification, risk stratification, and management of monoclonal gammopathy of undetermined significance: implications for recategorizing disease entities in the presence of evolving scientific evidence. Mayo Clin Proc, 85, 945-8.

Rajkumar SV, Dimopoulos MA, Palumbo A, et al (2014). International Myeloma Working Group updated criteria for the diagnosis of multiple myeloma. Lancet Oncol, 15, 

$538-48$.

Ruchirawat M, Settachan D, Navasumrit $P$, et al (2007). Assessment of potential cancer risk in children exposed to urban air pollution in Bangkok, Thailand. Toxicol Lett, 168, 200-9.

Sutandyo N, Firna E, Agustina J, et al (2015). Clinicopathology profile and bone involvement of multiple myeloma patients in dharmais national cancer hospital, Indonesia. Asian Pac J Cancer Prev, 16, 6261-5.

Tsai SS, Goggins WB, Chiu HF, et al (2003). Evidence for an association between air pollution and daily stroke admissions in Kaohsiung, Taiwan. Stroke, 34, 2612-6.

Yang Z, Harrison K, Park YA, et al (2007). Performance of the Sebia CAPILLARYS 2 for detection and immunotyping of serum monoclonal paraproteins. Am J Clin Pathol, 128, 293-9.

Yu XJ, Yang MJ, Zhou B, et al (2015). Characterization of somatic mutations in air pollution-related lung cancer. $E$ Bio $\mathrm{Med}, \mathbf{2}, 583-90$.

Winters N, Goldberg MS, Hystad $P$, et al (2015). Exposure to ambient air pollution in Canada and the risk of adult leukemia. Sci Total Environ, 526, 153-76. 